\title{
Erratum to: The Effects of Conventional and Non-conventional Processing on Glucosinolates and Its Derived Forms, Isothiocyanates: Extraction, Degradation, and Applications
}

\author{
Qian Deng ${ }^{1} \cdot$ Kyriaki G. Zinoviadou ${ }^{2}$ Charis M. Galanakis ${ }^{3}$ - Vibeke Orlien ${ }^{4}$. \\ Nabil Grimi $^{5} \cdot$ Eugène Vorobiev $^{5} \cdot$ Nikolai Lebovka $^{5,6} \cdot$ Francisco J. Barba $^{5,7}$
}

Published online: 31 March 2015

(C) Springer Science+Business Media New York 2015

\section{Erratum to: Food Eng Rev (2014) \\ DOI 10.1007/s12393-014-9104-9}

The authors would like to mention some references of the above-published article that are missing:

[145] Van Eylen D, Oey I, Hendrickx M, Van Loey A (2007) Kinetics of the stability of broccoli (Brassica oleracea cv. italica) myrosinase and

The online version of the original article can be found under doi:10.1007/s12393-014-9104-9.

Francisco J. Barba

francisco.barba@uv.es

1 Milne Fruit Products Inc., 804 Bennett Avenue, Prosser, WA 99350, USA

2 Department of Food Science and Technology, Perrotis College, American Farm School, 55102 Thessaloníki, Greece

3 Department of Research and Innovation, Galanakis Laboratories, Skalidi 34, 73131 Chania, Greece

4 Department of Food Science, Faculty of Science, University of Copenhagen, Rolighedsvej 30, 1958 Frederiksberg C, Denmark

5 Transformation Intégrée de la Matière Renouvelable (TIMR, EA 4297, UTC/ESCOM), Equipe Technologies Agroindustrielles, Centre de Recherche de Royallieu, Université de Technologie de Compiègne,

B.P. 20529-60205, Compiègne Cedex, France

6 Institute of Biocolloidal Chemistry (named after F.D. Ovcharenko), NAS of Ukraine, 42, blvr. Vernadskogo, Kiev 03142, Ukraine

7 Faculty of Pharmacy, Nutrition and Food Science Area, Universitat de València, Avda. Vicent Andrés Estellés, s/n, 46100 Burjassot, València, Spain isothiocyanates in broccoli juice during pressure/ temperature treatments. J Agric Food Chem 55:2163-2170

[146] Clariana M, Valverde J, Wijngaard H, Mullen AM, Marcos B (2011) High pressure processing of swede (Brassica napus): impact on quality properties. Innov Food Sci Emerg Technol 12:85-92

[147] Dai R, Lim L-T (2014) Release of allyl isothiocyanate from mustard seed meal powder. J Food Sci 79:E47-E53

Moreover, some references were incorrectly listed, and the numbering should be changed as follows:

1. Page 2, right column, "Indole GLs are derived from tryptophan and their content in some plants is rather high, such as Brussel sprout and broccoli [138]”.

The reference number should be [13].

2. In Page 6, it should be included: Adapted from [35]. In Fig. 4 An exemplary flow chart of "5Stages Universal Recovery Process" for the recovery of antioxidant compounds.

3. Page 9, right column, “... (Table 4) [51]”.

The reference number should be [52].

4. Pages 10-11, Table 4, reference numbers should be (from top to bottom):

[106], instead of [109].

[57], instead of [64].

[61], instead of [60].

[145], instead of [47].

[146], instead of [61].

[138] (no change). 
[66], instead of [72].

[65], instead of [71].

[54] (no change).

[52], instead of [51].

[37], instead of [36].

[38], instead of [37].

5. Page 12, under the figure, the note: "Fig. 5 ... Adapted with permission from [39]".

The reference number should be [37].

6. Page 12 , left column, “ $\ldots \mathrm{k}$ is the constant, and $\mathrm{t}$ is the treating time [57]".

The reference number should be [145].

7. Page 12, left column, "the authors also found that...first-order reaction model observed in red cabbage [57, 98]".

The reference numbers should be $[92,145]$.

8. Page 12, bottom of the left column, “...both HP and HT helped the deactivation of myrosinase [57]".

The reference number should be [145].
9. In page 12 , right column, second paragraph, “...of broccoli was still active at lower end of HP (100-500 MPa) [57]".

The reference number should be [145].

10. Page 13, left column, first paragraph, “...higher temperature $\left(40{ }^{\circ} \mathrm{C}\right)[60] "$.

The reference number should be [61].

11. Page 19, left column, 4th paragraph, "The enzymatic degradation of sinigrin...pungent sensation of mustard seed [110]".

The reference number should be [147].

12. Page 19, left column 4th paragraph, "Higher temperatures and higher relative humidity, ...AITC liberation from the mustard seed meal [110]".

The reference number should be [147].

Conflict of interest The authors declare that they have no conflict of interest. 